\title{
Commentary on the WHO classification of tumors of lymphoid tissues (2008): "Gray zone" lymphomas overlapping with Burkitt lymphoma or classical Hodgkin lymphoma
}

\author{
Robert P. Hasserjian • German Ott • \\ Kojo S. J. Elenitoba-Johnson • Olga Balague-Ponz • \\ Daphne de Jong • Laurence de Leval
}

Received: 17 May 2009 /Accepted: 19 May 2009/Published online: 27 June 2009

(C) Springer-Verlag 2009

\author{
Abstract The 2008 WHO Classification of Tumors of \\ Haematopoietic and Lymphoid Tissues has introduced two \\ new categories of high-grade B-cell lymphomas: entities in \\ which features of diffuse large B-cell lymphoma (DLBCL) \\ R. P. Hasserjian \\ Department of Pathology, \\ Massachusetts General Hospital and Harvard Medical School, \\ Boston, MA, USA \\ G. Ott \\ Department of Pathology, \\ Robert-Bosch-Krankenhaus and Institute of Clinical \\ Pharmacology, \\ Stuttgart, Germany \\ K. S. J. Elenitoba-Johnson \\ Department of Pathology, University of Michigan, \\ Ann Arbor, MI, USA \\ O. Balague-Ponz $\cdot$ D. de Jong \\ Department of Pathology, The Netherlands Cancer Institute, \\ Amsterdam, The Netherlands \\ L. de Leval \\ Department of Pathology, C.H.U Sart Tilman, \\ University of Liège, \\ Liège, Belgium \\ R. P. Hasserjian $(\varangle)$ \\ Pathology, Warren 2, Massachusetts General Hospital, \\ 55 Fruit Street, \\ Boston, MA 02114, USA \\ e-mail: rhasserjian@partners.org \\ L. de Leval $(\bowtie)$ \\ Department of Pathology, C.H.U. Sart Tilman, \\ Institute of Pathology, \\ B23, +1, 4000 Liège, Belgium \\ e-mail: L.deLeval@ulg.ac.be
}

overlap with Burkitt lymphoma (DLBCL/BL) or classical Hodgkin lymphoma (DLBCL/HL). The DLBCL/BL category encompasses cases that resemble Burkitt lymphoma morphologically, but have one or more immunophenotypic or molecular genetic deviations that would exclude it from the BL category; conversely, some cases have immunophenotypic and/or genetic features of BL, but display cytologic variability unacceptable for BL. Many of the cases in the DLBCL/BL category contain a translocation of $M Y C$ as well as either $B C L 2$ or $B C L 6$ (so-called double-hit lymphomas) and have a very aggressive clinical behavior. The DLBCL/HL category encompasses lymphomas that exhibit the morphology of classical Hodgkin lymphoma but the immunophenotype of DLBCL, or vice versa. Most DLBCL/HL cases described present as mediastinal masses, but this category is not limited to mediastinal lymphomas. These new categories acknowledge the increasing recognition of cases that display mixed features of two wellestablished diseases. Whether the existence of such cases reflects shortcomings of our current diagnostic armamentarium or a true disease continuum in which such hybrid or intermediate neoplasms actually exist remains to be determined.

Keywords Burkitt · Diffuse large B-cell lymphoma . Hodgkin lymphoma · Gray zone lymphoma

\section{Introduction}

Classification systems represent the language of both pathology and clinical medicine and provide a critical communication medium in establishing, applying, and 
validating therapeutic protocols. The 2008 WHO Classification of Tumors of Haematopoietic and Lymphoid Tissues has refined the 2001 Classification based on new and accumulated experimental evidence. Increasingly, diagnostic criteria are based on more diverse types of information than mere morphology, such as clinical information, immunophenotype, and (particularly in the 2008 Classification), molecular genetic studies [1]. However, this ever diversifying armamentarium of tools to interrogate neoplasms has an "Achilles Heel": tumors are inherently complex and do not necessarily provide predictable answers to our broad range of studies. These complexities can stymie our attempts to force individual cases into a particular category by several ways: the tumor may lie on a true biologic continuum between two entity definitions; it may exhibit an exceptional clinical behavior, morphology, immunophenotype, or genetic finding that mimics another tumor type; it may appear to change from one tumor type to another; or it may contain subclones that appear as two discrete tumors. In recognition of the inherent complexity of aggressive B-cell lymphomas, the WHO 2008 Classification has created two new categories for tumors that appear to lie on the continuum between diffuse large B-cell lymphoma (DLBCL) and Burkitt lymphoma (BL), and between DLBCL and classical Hodgkin lymphomas (CHL). While these new categories may prove to be frustrating to clinicians who wish to treat their patients for a familiar disease with a tested therapy, they acknowledge the current reality that optimal therapy for these overlapping entities is unclear. Moreover, the creation of these diagnostic categories maintains the "purity" of existing entities (DLBCL, BL, CHL), as the inclusion of borderline cases may compromise (and has most likely already compromised) the validity of studies that use looser inclusion criteria.

\section{Current "state of the art" of Burkitt lymphoma}

The 2008 WHO Classification continues to recognize three epidemiologic variants of BL (endemic BL, sporadic $\mathrm{BL}$, and immunodeficiency-associated $\mathrm{BL}$ ), but the previous atypical/Burkitt-like and plasmacytoid morphologic variants are no longer formally designated. Cases of $\mathrm{BL}$ that have features slightly deviating from the typical medium-sized monomorphous cytomorphology, such as increased nuclear irregularity, slight nuclear pleomorphism, and/or more prominent, single nucleoli (including most cases arising in adults) are classified simply as BL if they otherwise fit immunophenotypically and genetically. This change is supported by recent data showing that these morphologically "atypical" BL cases have a molecular signature similar to classic BL [2]. BL cases presenting as a leukemia without significant lymphadenopathy (previously known as 'L3' ALL) are retained within the BL category and represent the only recognized BL variant in the 2008 WHO Classification.

The characteristic immunophenotype of BL is that of strong CD10 expression, expression of BCL6, negativity for BCL2, and a Ki67 proliferation index (PI) of near 100\% (at least $90 \%$ of tumor cells) [3]. Immunohistochemistry for MYC protein is non-specific and is not useful for diagnostic purposes. The $M Y C$ rearrangement in $\mathrm{BL}$ usually occurs in a background of a relatively simple karyotype and should lack concomitant rearrangements of $B C L 2$ or BCL6 $[2,4]$. Two major recent gene expression profiling studies have shown that typical BL cases have a characteristic molecular signature $[2,5]$. Rare cases that are otherwise characteristic for $\mathrm{BL}$ lack a $M Y C$ translocation; the proportion of $\mathrm{BL}$ cases that are $M Y C$ negative is variable in published studies, likely reflecting differing pathologic inclusion criteria. These cases bear a molecular signature similar to BL cases that have rearranged $M Y C$, validating their classification as BL $[2,5]$. Recent data suggest that $\mathrm{BL}$ cases lacking a $M Y C$ rearrangement may upregulate MYC expression by alternative mechanisms, possibly involving the modulation of microRNAs [6].

\section{B-cell lymphoma unclassifiable, with features intermediate between diffuse large B-cell lymphoma and Burkitt lymphoma}

With the increasing use of detailed immunophenotyping panels and genetic studies, there has been increased recognition of cases that share many features with $\mathrm{BL}$, but differ with respect to one or more findings. There has been poor reproducibility when pathologists attempt to assign such cases to BL or DBLCL categories, even when integrating ancillary laboratory results such as cytogenetics and immunophenotype [7]. The molecular profiling study of Hummel et al. also identified a group of cases displaying a molecular signature intermediate between $\mathrm{BL}$ and DLBCL. Moreover, some cases classified by pathologists as DLBCL bore a molecular signature of BL. These findings suggest that a true continuum between these entities may exist $[2,5]$. Nevertheless, given the different therapies that are indicated, pathologists' hedging between $\mathrm{BL}$ and DLBCL is frustrating for clinicians and also affects the validity of clinical trials that aim to evaluate the effectiveness of therapies on discrete diagnostic entities. For these reasons, the WHO 2008 Classification established a new diagnostic category of B-cell lymphoma, unclassifiable, with features intermediate between DLBCL and BL (DLBCL/BL). These cases have morphologic, immunophenotypic, and genetic features that include aspects of both 
BL and DLBCL, and thus cannot be appropriately classified into either category.

DLBCL/BL cases consist largely of cases in which the pathologist considers a diagnosis of BL, but which are excluded from the category. Cases with an immunophenotype of BL (including a PI $>90 \%$ ) and with "starry-sky" appearance, but with marked variation in cytomorphology that is not acceptable for BL, are classified as DLBCL/BL. Conversely, cases that have morphology that is acceptable for BL (uniform or mildly pleomorphic medium-sized cells) may be placed into the DLBCL/BL category if they do not have the typical immunophenotype of BL; while occasional BL cases may have minor immunophenotypic deviations, such as weak positivity for BCL2, strong BCL2 staining, and/or a Ki67 proliferation index of $<90 \%$ are strong contraindications for a diagnosis of BL. Similarly, cases resembling $\mathrm{BL}$ with $M Y C$ rearrangements that are transformations of a documented prior low-grade B-cell lymphoma are classified as DLBCL/BL [8]. Rare cases resembling B-lymphoblastic leukemia/lymphoma (B-LBL) by morphology, TdT positivity, and negativity for surface immunoglobulin may bear a $M Y C$ rearrangement with or without a $B C L 2$ rearrangement $[9,10]$. It is controversial whether to diagnose these cases as B-LBL or as DLBCL/ BL. However, both of these entities are treated similarly with intensive chemotherapy regimens. Importantly, B-cell lymphomas composed of large cells or those with marked cellular pleomorphism should be classified as DLBCL irrespective of their $M Y C$ status, proliferation index, or immunophenotype: indeed, up to $15 \%$ of DLBCL cases bear a $M Y C$ translocation $[11,12]$ and some DLBCL cases may have proliferation indices approaching 100\% [13]. Although some of these cases have been shown to bear the molecular signature of $\mathrm{BL}$, using current diagnostic methods they must be classified as DLBCL.

When a $M Y C$ rearrangement is present in $\mathrm{DLBCL} / \mathrm{BL}$ cases, it often has atypical features, including one or more of the following: (1) rearrangement with a non- $I G$ partner; (2) as part of a complex karyotype; (3) concurrent rearrangements of BCL2 and/or BCL6 genes (so-called double-hit or triple-hit lymphoma lymphomas). "Doublehit" lymphomas with both $M Y C$ and $B C L 2$ rearrangements comprise a large and relatively well-characterized subset of DLBCL/BL. These cases exhibit a spectrum of morphology and a wide range of Ki67 proliferation index, but all strongly express BCL2 [14]. Rare cases of histologically low-grade follicular lymphoma that bear both $M Y C$ and $B C L 2$ rearrangements should not be placed into this category, as these are histologically distinct $[14,15]$. Rare cases of plasma cell myeloma may also bear a $M Y C$ or concurrent $M Y C$ and $B C L 2$ rearrangements and should not be included in the DLBCL/BL category $[14,16]$. Although gene expression profiling data is not available on DLBCL/
BL as a group, some "double-hit" cases with both $M Y C$ and $B C L 2$ rearrangement have been shown to have a molecular signature similar to classic $\mathrm{BL}$; other cases have a signature intermediate between BL and DLBCL [5]. Importantly, most cases of DLBCL with MYC translocation share the molecular signature of DLBCL, not BL [5].

The "double-hit" DLBCL/BL lymphomas are highly aggressive tumors that usually present with high stage of disease and appear to do poorly whether treated with intensive regimens used to treat BL or CHOP-like regimens $[13,17-19]$. The aggressive nature of these tumors likely reflects their simultaneous expression of both proproliferative (MYC) and anti-apoptotic (BCL2) oncoproteins. The clinical behavior of other cases fulfilling criteria for DLBCL/BL (such as cases with overlapping features of BL and DBLCL but lacking a "double-hit" or even a $M Y C$ rearrangement) is unknown. It is anticipated that DLBCL/ $\mathrm{BL}$ represents a biologically heterogeneous group that requires further genetic and clinical characterization.

\section{Questions and controversies}

What is the "gold standard" for a diagnosis of BL? The two recent gene expression profiling studies validated the genetic homogeneity of typical BL cases $[2,5]$. However, a substantial number of cases that would not be classified as BL (17-34\%, most likely including both DLBCL/BL and DLBCL cases according to 2008 WHO Classification criteria) had a molecular signature of BL. Cases with BL molecular signature did poorly as a group when treated with CHOP-like regimens. Conversely, BL and highproliferation DLBCL cases diagnosed by conventional criteria and treated with intensive regimens had similar outcome in one recent study [13]. Ultimately, the biologic "gold standard" of BL is that of an aggressive, but (with appropriately intensive chemotherapy) highly curable lymphoma. A prospective study is required to determine whether molecular signature, WHO classification, or a specific biologic marker (such as MYC translocation status or Ki67 proliferation index) most optimally identifies cases with this particular disease biology.

Should a MYC rearrangement be sought on all highly proliferative B-cell neoplasms? According to the 2008 WHO Classification, a diagnosis of BL can be made if the morphology and immunophenotype are perfect. In children, in whom classic morphology predominates, a MYC rearrangement is probably not necessary to confirm the diagnosis. Similarly, a case with overt DLBCL morphology does not require $M Y C$ investigation for classification, since DLBCL/BL would not be a diagnostic consideration (although it should be noted that MYC 
rearrangement is a poor prognostic feature in DLBCL with high proliferation index, and may provide helpful prognostic information in certain clinical contexts) [13]. Accordingly, it is probably prudent to assess for a $M Y C$ rearrangement in all adult patients in which $\mathrm{BL}$ or $\mathrm{BL} /$ DLBCL is a diagnostic consideration based on the morphology and immunophenotype.

When should BCL2 and BCL6 rearrangements be sought? Because the relative incidence of DLBCL/BL appears to increase with age, while that of BL decreases, it is probably prudent to examine any adult case of putative BL for concomitant BCL2 and BCL6 rearrangements that would argue against a diagnosis of BL. $M Y C$ rearranged cases with concomitant BCL2 or BCL6 rearrangements would be classified as DLBCL/BL, irrespective of the other features. These "double-hit" lymphomas have a much poorer prognosis than BL, even when treated with intensive chemotherapy regimens.

What about looking for complex karyotype and MYC rearrangement with non-IG partners? Different centers vary in the practice of obtaining cytogenetics on lymph node samples. Comparative genomic hybridization (CGH), that shows markedly different complexity patterns in $\mathrm{BL}$ and DLBCL, is not routinely available. Thus, while the presence of a known complex karyotype would tend to favor a diagnosis of $\mathrm{DLBCL} / \mathrm{BL}$ over $\mathrm{BL}$, practical limitations such as lack of suitable material for classical cytogenetics or non-availability of CGH (especially if only paraffin material is available) impede routine performance in many laboratories. Similarly, probes to assess the rearrangement of $M Y C$ with non- $I G$ gene loci are not widely available. Nevertheless, if performed, the knowledge that $M Y C$ is rearranged with a non- $I G$ locus may be helpful in assigning borderline cases (such as those with minor immunophenotypic or morphologic deviations from $\mathrm{BL})$ to the DLBCL/BL category.

\section{Primary mediastinal (thymic) large B-cell lymphoma and its borderlands with classical Hodgkin lymphoma}

Primary mediastinal large B-cell lymphoma (PMBL) was the first large B-cell lymphoma entity to be delineated, by virtue of its distinctive clinical and morphological features $[20,21]$. In contrast to other DLBCLs, PMBL tends to occur in younger patients, with a predilection for women, and usually presents as a localized, but often bulky anterior mediastinal mass with or without involvement of supraclavicular lymph nodes [20, 22]. Distinctive morphological features of PMBL include fine compartmentalizing sclerosis, the presence of cells with abundant clear cytoplasm and/or multilobated nuclei, and the presence of large ReedSternberg-like cells [23].

In the 20 years that have elapsed since the original descriptions of PMBL, further evidence has accumulated that supports the concept of a disease distinct from nonmediastinal DLBCL. The neoplastic B-cells express B-cell antigens, but often lack surface immunoglobulin (Ig), despite expression of the Ig-associated transcription factors BOB1, OCT2, and PU1 [24]. Expression of antigens related to the germinal center and post-germinal center stages of Bcell differentiation is variable, but most cases co-express BCL6 and MUM1/IRF4, often in the absence of CD10, suggesting an activated or post-geminal center phenotype $[24,25]$. The majority of tumors express CD30, usually heterogeneously and with weak to moderate intensity. The frequent expression of CD23 and MAL in PMBL has been taken as evidence to suggest its cellular derivation from an immunophenotypically similar peculiar population of "asteroid" B cells normally present in the thymic medulla [2628]. In distinction from other DLBCLs, expression of FIG1 (the product of the interleukin-4-induced gene 1) and TNFreceptor-associated factor 1 are characteristic of PMBL, especially when combined with nuclear c-REL localization [27, 29, 30]. Finally, the PMBL molecular signature differs from that of other DLBCLs [29, 31].

In practice, the diagnosis of $\mathrm{PMBL}$ requires the integration of the pathological and clinical presenting features. It must be stressed that the particular features listed above, albeit characteristic of PMBL, are not present in every case, and none is entirely specific. Moreover, among the different immunophenotypic markers that are differentially expressed in PMBL and other DLBCL, only CD23 and CD30 are available routinely in most diagnostic laboratories. In young patients with disease localized to the mediastinum (sometimes also involving supraclavicular nodes), histopathologic documentation of DLBCL is nearly always considered diagnostic of PMBL. In older patients, secondary mediastinal involvement by DLBCL is more common: in that setting, the characteristic features of PMBL should be demonstrated and it is preferable to restrict a PMBL diagnosis to cases that show the typical clinical and pathological features. Both entities are currently treated with anthracycline-based chemotherapy plus rituximab, and when adjusted for stage, have similar outcomes.

PMBL and nodular sclerosis CHL (NSHL) exhibit strikingly similar clinical presentations, as both diseases tend to occur as a localized mediastinal mass in young women. Moreover, in addition to some morphologic overlap (Reed-Sternberg-like cells and sclerosis), recent studies have documented many genetic and molecular similarities [29, 31]. However, importantly for diagnostic purposes, usual cases of PMBL and CHL also have several 
non-overlapping distinctive pathological features. The similarities and contrasting features of PMBL and $\mathrm{CHL}$ are summarized in Table 1. The diagnosis of NSHL requires a nodular growth pattern, broad bands of fibrosis, and lacunar variants of Reed-Sternberg cells that have a characteristic immunophenotype (CD45-, CD30+, CD15+/-). Unlike $\mathrm{CHL}$, the neoplastic cells of PMBL express CD45 and are characteristically CD15-. CD30 expression, when present, is typically weaker compared to the uniformly strong CD30 expression in CHL. Conversely, expression of B-cell associated antigens is strong and uniform in PMBL, and is usually weaker and more heterogeneous in the neoplastic cells of CHL. While Ig is not typically expressed in the neoplastic cells of either entity, Ig transcription factors such as OCT2 and BOB1 are maintained in PMBL, and may be absent in CHL. EBV may be present in CHL, but is absent in PMBL.

\section{B-cell lymphoma, unclassifiable, with features intermediate between diffuse large B-cell lymphoma and classical Hodgkin lymphoma}

Even after using differential diagnostic criteria, a small proportion of mediastinal large cell lymphomas cannot be classified. These cases, previously termed "gray zone lymphomas" or "large B-cell lymphomas with Hodgkin features" in the literature, represent a spectrum of tumors having characteristics of both PMBL and CHL [32-35]. In the 2008 WHO Classification, a novel category designated B-cell lymphoma, unclassifiable, with features intermediate between diffuse large B-cell lymphoma and Hodgkin lymphoma (DLBCL/HL) has been created to include these neoplasms [1]. DLBCL/HL mostly encompasses lymphomas with mediastinal presentation, but also includes occasional cases with features intermediate between

Table 1 Overlapping and contrasting features between primary mediastinal large B-cell lymphoma (PMBL) and mediastinal classical Hodgkin lymphoma, nodular sclerosis type (NSHL)

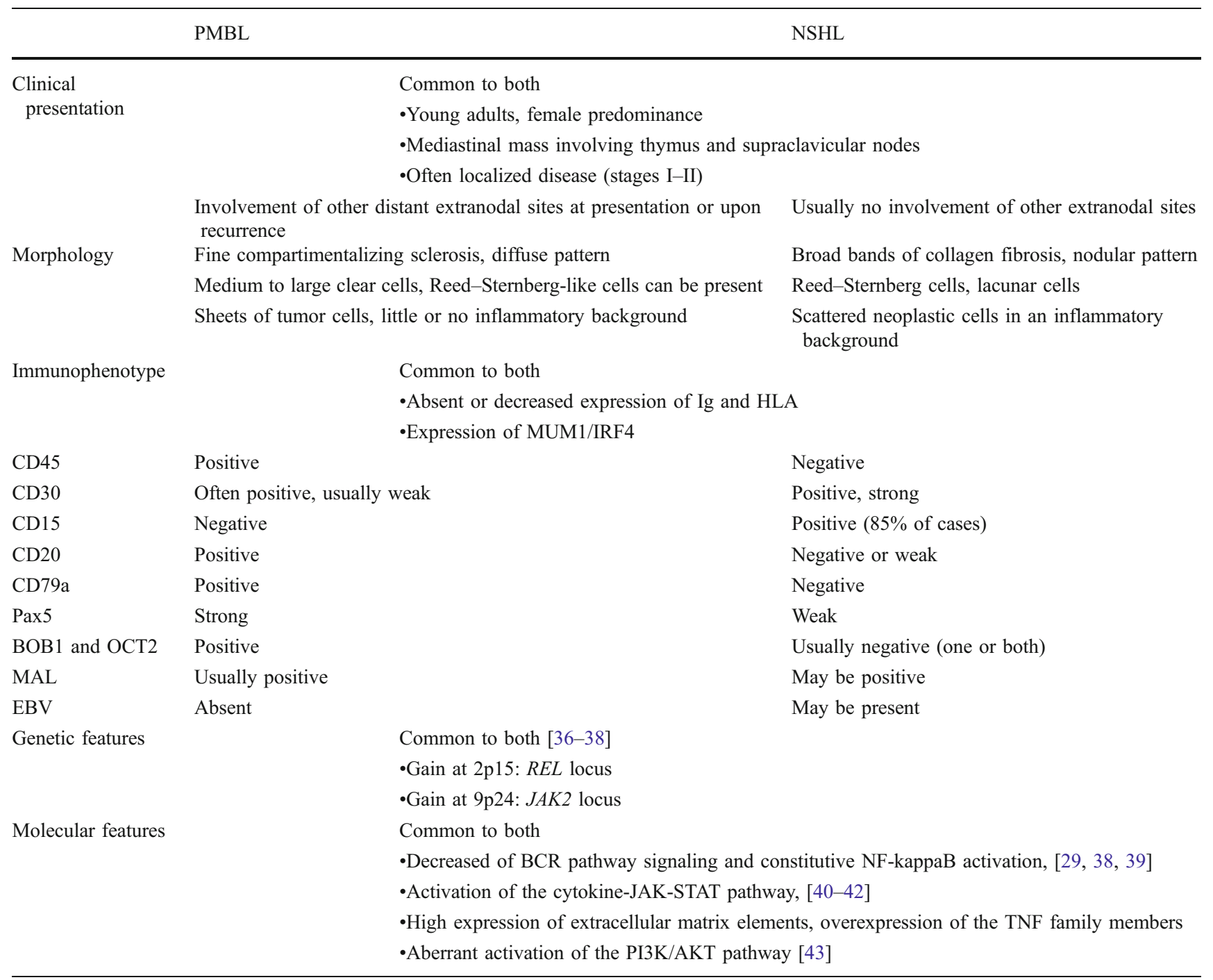


DLBCL and CHL involving non-mediastinal sites, provided all other possible diagnoses (in particular EBV-positive DLBCL of the elderly) have been excluded. For mediastinal tumors, such a diagnosis should be restricted to cases showing significant overlapping features, in particular marked diagnostic discordance between the morphology and immunophenotype. Features that would qualify a case as DLBCL/HL include: (1) strong expression of CD15 in a case otherwise resembling large B-cell lymphoma; (2) strong and diffuse expression of CD20 and/or other B-cell markers such as CD79a in a case morphologically suggestive of CHL but very rich in large neoplastic cells. Such cases support the existence of a spectrum of lymphomas that lie along a continuum between PMBL and CHL [35] and further validate the demonstrated molecular link between these entities. PMBL and CHL may also coexist in some patients as simultaneous composite lesions or as sequential tumors, the typical scenario being $\mathrm{CHL}$ occurring first, followed by PMBL within the first few years [32, 35]. A clonal relationship has been demonstrated in some of such sequential cases. The clinical relevance of this "unclassifiable" category is uncertain, but it has been suggested that the clinical outcome of DLBCL/HL cases may be less favorable than that of either PMBL or CHL. There is currently no consensus on treatment for this entity, although some groups have recommended treating these cases as aggressive B-cell lymphomas [35].

These "borderline cases" are always diagnostically difficult; workup of such cases should include most of the markers suggested in Table 1 (including the B-cell transcription factors OCT2 and BOB.1). While there is no current precise recommendation regarding the weight to put on each specific immunostain, there is general agreement that DLBCL/HL includes cases in which the immunoprofile is markedly different from that expected from the morphology.

\section{Conclusion}

The designation of these two new "gray zone" case groups as formal diagnostic entities has taken some pressure off pathologists who previously felt compelled to classify the unclassifiable. However, one potential concern is that these new diagnoses may attract cases that display only minor diversity within the established entities of BL, DLBCL, and CHL. To avoid this, it is important that the diagnostic spectra of BL, DLBCL, and CHL be clearly defined so that only the truly intermediate or hybrid cases are included within the new entities. Also, we must acknowledge and accept that these intermediate categories do not represent single diseases: they likely encompass multiple biologic entities or, alternatively, may represent a mixture of cases of each "parent" entity that we do not yet know how to properly assign. The true "gold standard" that defines even well-known neoplasms such as BL or MLBL is as yet unknown. Perhaps with further study, particular markers may be discovered that will allow assignment of most of these intermediate cases to discrete diagnostic groups with predictable clinical behavior and optimized therapy. Until then, these two new diagnostic categories provide a forum to study and better understand the biology behind these complex and aggressive lymphoid neoplasms.

\section{References}

1. Swerdlow S, Campo E, Harris NL, Jaffe ES, Pileri SA, Stein H, Thiele J, Vardiman JW (2008). WHO Classification of tumors of Hematopoietic and Lymphoid Tissues. IARC, Lyon

2. Hummel M, Bentink S, Berger $\mathrm{H}$ et al (2006) A biologic definition of Burkitt's lymphoma from transcriptional and genomic profiling. N Engl J Med 354(23):2419-2430

3. Dogan A, Bagdi E, Munson P, Isaacson P (2000) CD10 and bcl-6 expression in paraffin sections of normal lymphoid tissue and Bcell lymphomas. Am J Surg Pathol 24:846-852

4. Boerma EG SR, Kluin PM, Baudis M (2009) Translocations involving $8 \mathrm{q} 24$ in Burkitt lymphoma and other malignant lymphomas: a historical review of cytogenetics in the light of todays knowledge Leukemia 23(2):225-234

5. Dave SS, Fu K, Wright GW et al (2006) Molecular diagnosis of Burkitt's lymphoma. N Engl J Med 354(23):2431-2442

6. Leucci E, Cocco M, Onnis A et al (2008) MYC translocationnegative classical Burkitt lymphoma cases: an alternative pathogenetic mechanism involving miRNA deregulation. J Pathol 216 (4): $440-450$

7. Haralambieva E, Boerma EJ, van Imhoff GW et al (2005) Clinical, immunophenotypic, and genetic analysis of adult lymphomas with morphologic features of Burkitt lymphoma. Am J Surg Pathol 29 (8): 1086-1094

8. Leoncini L, Delsol G, Gascoyne RD et al (2005) Aggressive Bcell lymphomas: a review based on the workshop of the XI Meeting of the European Association for Haematopathology. Histopathology 46(3):241-255

9. Thangavelu M, Olopade O, Beckman E et al (1990) Clinical, morphologic, and cytogenetic characteristics of patients with lymphoid malignancies characterized by both $\mathrm{t}(14 ; 18)(\mathrm{q} 32 ; \mathrm{q} 21)$ and $\mathrm{t}(8 ; 14)(\mathrm{q} 24 ; \mathrm{q} 32)$ or $\mathrm{t}(8 ; 22)(\mathrm{q} 24 ; \mathrm{q} 11)$. Genes Chromosomes Cancer 2(2): 147-158

10. Nakamura F, Tatsumi E, Tani K et al (1996) Coexpression of cellsurface immunoglobulin (sIg), terminal deoxynucleotidyl transferase (TdT) and recombination activating gene 1 (RAG-1): two cases and derived cell lines. Leukemia 10(7):1159-1163

11. van Imhoff GW, Boerma EJ, van der Holt B et al (2006) Prognostic impact of germinal center-associated proteins and chromosomal breakpoints in poor-risk diffuse large B-cell lymphoma. J Clin Oncol 24(25):4135-4142

12. Cigudosa JC, Parsa NZ, Louie DC et al (1999) Cytogenetic analysis of 363 consecutively ascertained diffuse large B-cell lymphomas. Genes Chromosomes Cancer 25(2):123-133

13. Mead GM, Barrans SL, Qian W et al (2008) A prospective clinicopathologic study of dose-modified CODOX-M/IVAC in patients with sporadic Burkitt lymphoma defined using cytogenetic and immunophenotypic criteria (MRC/NCRI LY10 trial). Blood 112(6):2248-2260 
14. Kanungo A, Medeiros LJ, Abruzzo LV, Lin P (2006) Lymphoid neoplasms associated with concurrent $\mathrm{t}(14 ; 18)$ and $8 \mathrm{q} 24 / \mathrm{c}-\mathrm{MYC}$ translocation generally have a poor prognosis. Mod Pathol 19 (1):25-33

15. Hasserjian RP SR, Turbiner J, Wang S, Pinkus GD, Harris NL, Iafrate AJ (2008) BCL2, BCL6, and MYC rearrangements in follicular lymohoma: comparison of FISH and immunohistochemical results on a tissue microarray and correlation with patient outcome. Mod Pathol 21(Suppl 1):256A

16. Avet-Loiseau H, Gerson F, Magrangeas F, Minvielle S, Harousseau JL, Bataille R (2001) Rearrangements of the c-myc oncogene are present in $15 \%$ of primary human multiple myeloma tumors. Blood 98(10):3082-3086

17. Macpherson N, Lesack D, Klasa R et al (1999) Small noncleaved, non-Burkitt's (Burkitt-Like) lymphoma: cytogenetics predict outcome and reflect clinical presentation. J Clin Oncol 17 (5):1558-1567

18. Kolman OK, Snuderl M, Ferry JA, Hochberg EP, Chen YB, Hasserjian RP, Rahemtullah A (2008) Clinicopathologic features of B-cell lymphomas with concurrent BCL-2 and c-MYC gene rearrangements. Mod Pathol 21(Suppl 1):260A

19. McClure RF, Remstein ED, Macon WR et al (2005) Adult B-cell lymphomas with Burkitt-like morphology are phenotypically and genotypically heterogeneous with aggressive clinical behavior. Am J Surg Pathol 29(12):1652-1660

20. Jacobson J, Aisenberg A, Lamarre L et al (1988) Mediastinal large cell lymphoma: an uncommon subset of adult lymphoma curable with combined modality therapy. Cancer 62:1893-1898

21. Addis BJ, Isaacson PG (1986) Large cell lymphoma of the mediastinum: a B-cell tumour of probable thymic origin. Histopathology 10(4):379-390

22. Cazals-Hatem D, Lepage E, Brice P et al (1996) Primary mediastinal large B-cell lymphoma. A clinicopathologic study of 141 cases compared with 916 nonmediastinal large B-cell lymphomas, a GELA ("Groupe d'Etude des Lymphomes de l'Adulte") study. Am J Surg Pathol 20(7):877-888

23. Lamarre L, Jacobson JO, Aisenberg AC, Harris NL (1989) Primary large cell lymphoma of the mediastinum. A histologic and immunophenotypic study of 29 cases. Am J Surg Pathol 13 (9):730-739

24. Pileri SA, Gaidano G, Zinzani PL et al (2003) Primary mediastinal B-cell lymphoma: high frequency of BCL-6 mutations and consistent expression of the transcription factors OCT-2, BOB.1, and PU.1 in the absence of immunoglobulins. Am J Pathol 162 (1):243-253

25. de Leval L, Ferry JA, Falini B, Shipp M, Harris NL (2001) Expression of bcl-6 and CD10 in primary mediastinal large B-cell lymphoma: evidence for derivation from germinal center B cells? Am J Surg Pathol 25(10):1277-1282

26. Copie-Bergman C, Plonquet A, Alonso MA et al (2002) MAL expression in lymphoid cells: further evidence for MAL as a distinct molecular marker of primary mediastinal large B-cell lymphomas. Mod Pathol 15(11):1172-1180

27. Copie-Bergman C, Boulland ML, Dehoulle C et al (2003) Interleukin 4-induced gene 1 is activated in primary mediastinal large B-cell lymphoma. Blood 101(7):2756-2761

28. Calaminici M, Piper K, Lee AM, Norton AJ (2004) CD23 expression in mediastinal large B-cell lymphomas. Histopathology 45(6):619-624
29. Savage KJ, Monti S, Kutok JL, et al (2003) The molecular signature of mediastinal large B-cell lymphoma differs from that of other diffuse large B-cell lymphomas and shares features with classical Hodgkin's lymphoma. Blood 102(12):3871-3879

30. Rodig SJ, Savage KJ, LaCasce AS et al (2007) Expression of TRAF1 and nuclear c-Rel distinguishes primary mediastinal large cell lymphoma from other types of diffuse large B-cell lymphoma. Am J Surg Pathol 31(1):106-112

31. Rosenwald A, Wright G, Leroy K et al (2003) Molecular diagnosis of primary mediastinal $\mathrm{B}$ cell lymphoma identifies a clinically favorable subgroup of diffuse large B cell lymphoma related to Hodgkin lymphoma. J Exp Med 198(6):851-862

32. Rudiger T, Jaffe ES, Delsol G et al (1998) Workshop report on Hodgkin's disease and related diseases ('grey zone' lymphoma). Ann Oncol 9(Suppl 5):S31-38

33. Calvo KR, Traverse-Glehen A, Pittaluga S, Jaffe ES (2004) Molecular profiling provides evidence of primary mediastinal large B-cell lymphoma as a distinct entity related to classic Hodgkin lymphoma: implications for mediastinal gray zone lymphomas as an intermediate form of B-cell lymphoma. Adv Anat Pathol 11(5):227-238

34. Garcia JF, Mollejo M, Fraga M et al (2005) Large B-cell lymphoma with Hodgkin's features. Histopathology 47(1):101-110

35. Traverse-Glehen A, Pittaluga S, Gaulard P et al (2005) Mediastinal gray zone lymphoma: the missing link between classic Hodgkin's lymphoma and mediastinal large B-cell lymphoma. Am J Surg Pathol 29(11):1411-1421

36. Joos S, Otano-Joos MI, Ziegler S et al (1996) Primary mediastinal (thymic) B-cell lymphoma is characterized by gains of chromosomal material including $9 \mathrm{p}$ and amplification of the REL gene. Blood 87(4):1571-1578

37. Joos S, Granzow M, Holtgreve-Grez H et al (2003) Hodgkin's lymphoma cell lines are characterized by frequent aberrations on chromosomes $2 p$ and $9 p$ including REL and JAK2. Int J Cancer 103(4):489-495

38. Wessendorf S, Barth TF, Viardot A et al (2007) Further delineation of chromosomal consensus regions in primary mediastinal B-cell lymphomas: an analysis of 37 tumor samples using high-resolution genomic profiling (array-CGH). Leukemia 21(12):2463-2469

39. Feuerhake F, Kutok JL, Monti S et al (2005) NFkB activity, function, and target-gene signatures in primary mediastinal large B-cell lymphoma and diffuse large B-cell lymphoma subtypes. Blood 106(4):1392-1399

40. Guiter C, Dusanter-Fourt I, Copie-Bergman C et al (2004) Constitutive STAT6 activation in primary mediastinal large Bcell lymphoma. Blood 104(2):543-549

41. Melzner I, Bucur AJ, Bruderlein S et al (2005) Biallelic mutation of SOCS-1 impairs JAK2 degradation and sustains phospho-JAK2 action in the MedB-1 mediastinal lymphoma line. Blood 105 (6):2535-2542

42. Weniger MA, Melzner I, Menz CK et al (2006) Mutations of the tumor suppressor gene SOCS-1 in classical Hodgkin lymphoma are frequent and associated with nuclear phospho-STAT5 accumulation. Oncogene 25(18):2679-2684

43. Renne C, Willenbrock K, Martin-Subero JI et al (2007) High expression of several tyrosine kinases and activation of the PI3K/ AKT pathway in mediastinal large B cell lymphoma reveals further similarities to Hodgkin lymphoma. Leukemia 21(4):780-787 